\title{
Large Scale Design and Management of Ecological Region Based on IoT
}

\author{
Danyan Zhang \\ Tianjin Electronic Information College, Tianjin, 300350, China
}

\begin{abstract}
Keywords: Design and management, Ecological region, Internet of things
\end{abstract}
\begin{abstract}
The planning and management of ecological region is the action of design and operation to transform the economic structure and growth model through the problem-solving of environmental pollution and ecological destruction based on the concept regional sustainable development. Under the background of current information era and combined with the Internet of things technology, this paper explores the application of IoT in ecological function division, environmental monitoring, ecological data management and ecological environment improvement to provide some references for the relative researchers.
\end{abstract}

\section{Introduction}

With the global ecological environment problems intensified, ecological planning is becoming more and more attention and gradually become an important way to achieve sustainable development. The goal is to enhance the ability of regional sustainable development. From the perspective of regional ecological function, characteristics of regional resources and social economic conditions, reasonable planning and development way and the social economic development and utilization of resources, environmental protection and natural system in regional development and economic development, so as to achieve the virtuous cycle of resource utilization, environmental protection and economic growth, and continuously improve the ability of sustainable development region. Ecological region development planning is a regional sustainable development based on local resources, solve the problem of environmental pollution and ecological damage in the development of the economy and carry out the transformation of the economic structure and growth mode planning. The emergence and development of ecological planning reflects the human understanding of nature and the change of the way of dealing with nature. Ecological planning is becoming an effective tool to solve the sustainable development of social economy. The Internet of things has caused a new revolution of information technology, the user terminal extends to any object of life, to the local area network and Internet based terminal equipment, personnel and objects interconnection, automation and intelligent management, planning and management of ecological region is one of the key areas for the application of networking technology. With the development of the times, the Internet of things technology has been applied in more and more fields, and has brought profound changes in these areas, and constantly make new breakthroughs, can be described as changing rapidly. Ecological regional planning and management of Internet of things technology is the trend. Therefore, it is necessary to study the application of Internet of things in the planning and management of ecological region.

\section{Brief Introduction of IoT Technology}

The use of a new generation of networking technologies, such as radio frequency identification technology, sensor technology, super computer technology, cloud computing and other sensors embedded or equipment contribute the Internet of Things in the application of railways, highways, bridges, buildings, and other objects in the water supply system, and through the formation of interconnection Internet of things by supercomputers and cloud computing technology, analysis and processing of massive data and information networking. It will integrate the implementation of intelligent control and management, so as to achieve the wisdom of the world, finally realizes the Internet of things wisdom of the earth. The Internet is the development of technology and Internet 
technology based on network, is based on the RFID technology, computer network technology, wireless communication technology, the huge network consists of a large number of Internet readers and numerous mobile electronic tags. This definition is the protagonist of the technology, through the technology, give each item a "identity card" electronic tag reader can automatically and real-time of articles for recognition, positioning, tracking, monitoring and triggering the corresponding event. The concept of Internet of things presents the development of technology and Internet technology. This technology as the main body to explain the theory of the Internet of things is the theory of our country in the early days of the general understanding of the Internet of things. Things in a narrow sense, is connected to the network of goods items, intelligent identification and management to achieve the objects in a broad sense, is a kind of ubiquitous, things and things, between people and the information networks, can breed all kinds of new applications and services.

\section{Applications of IoT in the Large Scale Design and Management of Ecological Region}

Ecological Functions Division. Ecological function is the ability to determine or influence the natural productivity to maintain the existence and development of the system, also refers to the ecosystem and ecological process formed by natural environment condition and effectiveness of human survival and maintenance. The understanding of ecological function of human beings has gone through a long process of subject synthesis and knowledge accumulation. Processing capacity is the ecological system of production function refers to of material and energy regulating function is the embodiment of ecosystem is an ecosystem and ecological process of natural environment and effect of human survival and maintenance for interference or disaster resistance service function. It not only provides food, medicine and other raw materials for the production of human life, more important is to maintain the survival of the human life support system, life sustaining material biogeochemical cycle and hydrological cycle, maintaining the diversity of species, purify the environment, maintain the balance and stability of atmospheric chemistry. But people gradually realize that the ecosystem service function is the basis for human survival and modern civilization, is the cultural environment, good natural scenery and recreation environment, excellent characteristics of ecological quality, but high sensitivity, increase ecological system instability. How to objectively evaluate the ecosystem service function needs to be further studied. The environmental protection department due to technical limitations, do not collect real-time emission data, or difficult to find evidence of illegal enterprises, but also the lack of effective means of monitoring and Analysis on pollution situation of overall regional ecological environment. The arrival of the era of Internet of things and big data, it will provide important technical means for environmental law enforcement, through the establishment of intelligent environmental enforcement system, to achieve real-time, continuous and extensive monitoring of environmental pollution. Ecological function regionalization is defined according to the regional ecological environment factors and ecological service function of space law, divides the area into different ecological function areas, rational distribution of industrial and agricultural production, provide the basis for the protection of the regional ecological environment. Ecological function regionalization is outlined from the changes of regional land use status and characteristics of the ecological environment on the macro level, combination of the characteristics and laws of the correct understanding of ecological system for regional management departments, provide the basis for reasonable development and protection of regional resources.

Ecological Environment Monitoring. Water quality monitoring is closely related to people's lives, for the protection of water resources, development and prevention provides basis: managers can analyze the source of pollution, promptly shut down some heavy polluting enterprises ", rectify some discharge standards of the enterprise, as the water is important for human beings than any other material. many areas of serious water shortage, water quality and ecological environment monitoring is particularly important, in addition to the monitoring of contaminated areas, but also seriously protect non polluted rivers, pollution control. The main detection methods in China are water monitoring and pollution monitoring. Based on the technology of Internet of things, the electronic sensor is placed directly in the water. The main object of pollution monitoring is industrial waste 
water, electronic sensors can be placed in the discharge port, once the drainage does not meet the standard, it will be passed to the computer, notify the management personnel in a timely manner. China's urbanization process is accelerating, there is a series of environmental problems, the ecological environment of the main problem is that in the past, the traditional means of monitoring by specialized personnel to the scene of sampling, laboratory testing, monitoring report takes a few days to get, this method cannot deal with the sewage in the process of urbanization. In today's world, the impact of ecological damage is not limited to a country or a region, but will affect a number of countries and regions. Therefore, it is also necessary to cooperate with the global sensor data interoperability, the international environment to achieve common governance. All of the above data, through the Internet for real-time collection, summary to the corresponding data center, the formation of large data ecology, to provide effective data support for ecological data the next step of the analysis and management of ecological environment planning. As the detection time is relatively long, the human cost is relatively high, and the detection cycle is relatively long, cannot do real-time detection, the effect of the best time to solve the problem of pollution: networking technology provides a good solution to this problem, the real-time monitoring can reduce the difficulty of supervision of staff, reduce the randomness of pollution detection the increase of scientific and accurate monitoring, and ultimately improve the sewage treatment effect.

Ecological Data Management. The concept of big data is not limited to a large amount of data, but also to deal with massive data mining and processing of mining solutions. How to store, classify, excavate and visualize the large ecological data is also a technical problem to be considered in the construction of ecological civilization. In the same way, it can be considered from individuals, families, communities, businesses, regions, countries and international levels. The individual and household level data can be stored in a private cloud, the consumption of resources mainly mining individuals and families, individuals and families to cultivate awareness of ecological civilization; the community level data can be the establishment of small and medium sized enterprise cloud storage, the main energy source mining community resources consumption, provide the basis for the establishment of scientific and reasonable fees the enterprise level data can be established; large and medium-sized enterprise cloud storage, the main mining enterprise pollution rules and provide scientific data for enterprises using waste; regional and national level data can create a large enterprise of cloud storage, comprehensive analysis, dig out the changes of the ecological environment in different periods in different regions of the offer the basis for the government to make plans for the construction of ecological civilization, scientific and reasonable. Of course, these multi-level data is not isolated, but the organic connection, unified mining, hierarchical expression. The integration of personal data will rise to the national level, in addition, the national level of data processing results can also be expressed to individuals. Networking data can make us very rich ecological environmental data collected, and largely exclude subjective factors, to further improve the data accuracy and reliability, and can achieve real-time collection of data. Combined with the thinking and methods of big data, ecological environmental science research will enter into a new era.

Ecological Environment Improvement. Building a resource-saving society is an important part of ecological civilization construction in china. We can realize the sustainable development of China rely on science and technology, relying on science and technology to form less input and more output production and less emission, the consumption pattern of a new road to industrialization and urbanization, the development of production, affluent life and so on. Internet of things and big data can not only achieve the ecological environment monitoring and analysis, but also can be used to protect and improve the ecological environment. The networking and big data can show all kinds of human activities on the ecological environment caused by positive or negative effects of more fully image to people, cultivate personal, collective and social participation in the construction of ecological civilization consciousness. If there is no large data networking and participation, individual impacts on ecological environment caused by it will be difficult to assess, resulting in a lot of people think that environmental problems are caused by other people or other businesses, and their daily activities have no relationship. Therefore, we will generally believe that environmental protection is a matter of others or the environmental protection department, the individual's 
awareness of environmental protection is weak. And through the Internet of things and big data, will be able to quantify the impact on the ecological environment of individuals, and in accordance with the individual activities of the vivid image of the tips, which is conducive to improving the personal awareness of environmental protection. The Internet of things and the ecological big data can also be the main body of society as a whole, through collaboration and cooperation between the various subjects, the whole society to participate in the cultivation of ecological civilization awareness. Ecology is an organic whole which is interconnected and influenced by many subsystems, and the ecological environment in different regions and different periods is mutual influence. How to improve the effect of the state and the government adopted the policy of ecological protection and environmental protection measures on the ecological environment, which needs to be evaluated in a global and a relatively long period of time. Through networking and big data can provide a means of analysis of the monitoring results of whole area, multi temporal and multi levels, using the results of the analysis of these rich monitoring, can be more scientific and reasonable evaluation of the effect of the construction of ecological civilization. To improve the ecological environment in the future, we need to apply modern science and technology civilization, and build a strong support for the construction of ecological civilization.

\section{Conclusion}

With the development of information technology and social economy, people pay more attention to the construction of ecological environment. The technology of Internet of things is rapidly applied to the construction of ecological region. It can not only help the planners to complete the function design of ecological region, but also can monitor the ecological environment and manage the ecological data. On this basis, the Internet of things can improve people's behavior habits to improve the ecological environment quality.

\section{References}

[1] Li Xiaoxue, The Internet of Things Technology in the Application of Ecological Environmental Monitoring Analysis [J]. New Technology \& New Process, 2015(8): 72-75.

[2] Zhang Wanliang, Development of marine regional ecological monitoring system based on the internet of things [J]. Ship Science and Technology, 2017, 39(2A): 139-141.

[3] Wang Xijie, Application research of ecological environment monitoring based on internet of things technology [J]. Transducer and Microsystem Technologies, 2011, 30(7): 149-152.

[4] Du Keming, Chu Jinxiang, Sun Zhongfu, Zheng Feixiang, Xia Yu, Yang Xiaodong. Design and implementation of monitoring system for agricultural environment based on Web GIS with Internet of Things [J]. Transactions of the Chinese Society of Agricultural Engineering, 2016, 32(4): 171-178.

The Construction of Smart City Based on the Internet of Things

[5] Hao Shuchi, The Construction of Smart City Based on the Internet of Things [J]. Urban Insight, 2012(4): 62-67.

[6] Yunhe Pana, Yun Tiana, Xiaolong Liua, Dedao Gud, Gang Huad, Urban Big Data and the Development of City Intelligence [J].Engineering, 2016(2): 171-178. 